BMJ Open Sport \& Exercise Medicine

\section{Preparticipation medical evaluation for elite athletes: EFSMA recommendations on standardised preparticipation evaluation form in European countries}

To cite: Ionescu AM, Pitsiladis YP, Rozenstoka S, et al. Preparticipation medical evaluation for elite athletes: EFSMA recommendations on standardised preparticipation evaluation form in European countries. BMJ Open Sport \& Exercise Medicine 2021;7:e001178. doi:10.1136/ bmjsem-2021-001178

- Additional supplemental material is published online only. To view, please visit the journal online (http://dx.doi. org/10.1136/bmjsem-2021 001178).

Accepted 2 October 2021
Check for updates

(C) Author(s) (or their employer(s)) 2021. Re-use permitted under CC BY-NC. No commercial re-use. See rights and permissions. Published by BMJ.

For numbered affiliations see end of article.

Correspondence to Dr Alina Maria Smaranda; alina.smaranda@drd.umfcd.ro

\section{ABSTRACT}

Sports medicine is a medical specialty that supports the performance of professional and amateur athletes while maintaining their health. Sports medicine professionals need to ensure the safe participation of athletes in sports activities achieved through a periodical preparticipation evaluation (PPE) and a regular medical monitoring of the athletes' health in accordance with the latest recommendations regarding health condition and medical history, physical working capacity, training period and programme, recovery, nutrition, use of supplements, injuries prevention and safe return to play. In order to harmonise these national variations in the content and application of the PPE, the EFSMA Scientific and Educational Commission proposes a 'gold standard' for elite athletes across Europe.

Important objectives of PPE are early detection and prevention of severe complications during sports activities both in leisure time and competitive sports. The PPE should entail the following diagnostic components: health status, anthropometry, functional and exercise capacity. It is of utmost importance to develop and implement preventive strategies such as the PPE. Besides monitoring the health status of athletes, the PPE plays an important role in the selection process, bringing valuable information for coaches and supporting a personalised treatment approach. Screening of athletes through a standardised digital PPE could be beneficial for a better understanding of the impact of long-term physical activity. Furthermore, PPE leads the scientific community to a way of working closer together in the interest of the athletes.

\section{BACKGROUND}

Sports and Exercise Medicine (SEM) is a multidisciplinary, clinical and academic medical specialty that supports the performance of athletes while maintaining their health. SEM professionals need to ensure the safe participation of athletes in sports activities achieved through a periodical medical preparticipation evaluation (PPE) and regular medical

\section{Key points}

Implementing a standardised preparticipation evaluation across Europe in order to provide the best care to all elite athletes.

- Emphasising the importance of ECG screening among athlete population.

- Pointing out the necessity of training physicians into interpretation of athletes' ECG.

- Setting the basis for a future common, digita database.

monitoring of the athletes' health for identification of medical problems with possible life-threatening complications due to inherited or acquired conditions. The goals of the PPE also include fitness assessment, prescription of recovery and nutritional strategies, injuries prevention and safe return to play.

In 2018 Ron Golan (Israel) and Petra Zuped (Slovenia) applied a questionnaire to evaluate the current status of the PPE in Europe. The overview of the results obtained from 30 European countries emphasised that although PPE is widely accepted, its use and content are not standardised and important variations in terms of the different categories of athletes subjected to it (ie, professionals; elite or junior athletes licensed by Sport Federations or by the National Olympic Committee), legal aspects around it (eg, PPE compulsory or not), and the content and the cost of PPE. The results were presented through descriptive statistics. (11th EFSMA Congress of Sports Medicine, Portoroz, Slovenia, 2019, www.efsma.org.)

In most investigated European countries, PPE is mandatory only for competitive sports $(21 / 30=70 \%)$, and in nine countries, it is strongly recommended. In 24 countries, SEM specialists are qualified to conduct the PPEs. 
In other countries, the doctors conducting the PPEs are certified SEM physicians or team physicians with relevant certification and additional education in ECG and cardiopulmonary exercise testing.

To harmonise these national variations in the content and application of the PPE, the EFSMA Scientific and Educational Commission proposes a 'gold standard' for elite athletes across Europe. The proposed protocol is a basis for a comprehensive medical screening (https:// www.efsma.org/newsevents/news-events/251-efsma-preparticipation-evaluations-ppe- forms.html). ${ }^{1}$

The objectives of the current paper are to emphasise the need for a standardised PPE among European countries, to raise awareness of the importance of ECG screening during PPE, to recommend a comprehensive PPE form based on three diagnostic pillars and to create the framework for strategic planning and policy development within Europe. The target group is represented by elite athletes who could benefit from implementing a homogenous medical screening protocol, such as the EFSMA PPE form. There is a strong belief that if all countries performed the PPE using the same template, SEM physicians would improve their standard of care, would have a broader picture of the athletes' health issues and nonetheless would benefit from emerging prospective studies.

\section{WHY IS PPE NEEDED?}

The main scope of the PPE is protecting the health of the athletes. First and importantly PPE objectives are early detection and prevention of severe or threatening complications during sports activities both in leisure time and competitive sports. ${ }^{2-5}$ The PPE is a key tool in the process of screening for conditions that may be life-threatening, disabling or may predispose to injury or illness. Secondary, PPE is useful to determine and improve the level of performance, to help the athlete's staff (coach, athletic trainer, strength and conditioning trainer, physiotherapist, etc) in the selection process and further optimise the training according to the health status, to counsel the athlete regarding health issue and nonetheless to establish a relationship between SEM physician and athlete.

One of the longstanding debates is the question of including ECG screening in the PPE for all competitive athletes. ECG at rest as a mandatory examination in athletes is strongly recommended in most European countries. As such, there is long-standing controversy about whether ECG at rest should also be performed in all athletes, whereas in the USA and Canada is not required for all. Main arguments against ECG are the lack of large prospective studies with hard endpoints such as cardiac events or mortality. Some authors within former years emphasise the low sensitivity, specificity and predictive values of the ECG. ${ }^{6-8}$ However, current studies with newer criteria and interpretation increased the validity of ECG, as false positive and negative findings decreased significantly (four scientific
Box 1 Inherited diseases with life-threatening risk of cardiac arrest ${ }^{2}$

Channelopathies or 'electrical' conduction diseases

- Long-QT-syndrome, short-QT-syndrome, Brugada-syndrome.

- Catecholaminergic polymorphic ventricular tachycardia (CPVT) cardiac conduction diseases.

- Pre-excitation and conduction abnormalities (eg, WPW syndrome with atrial fibrillation).

Cardiac structural diseases

- Hypertrophic cardiomyopathy with or without outflow obstruction dilated cardiomyopathy (familial).

- Arrhythmogenic right ventricular dysplasia (or cardiomyopathy) left ventricular non-compaction cardiomyopathy.

Others

- Marfan-syndrome (especially in tall athletes, eg, basketball, volleyball, rowing).

Coronary artery anomalies (eg, abnormal origin or bridging).

articles from 2013 published by Drezner et al).$^{9-12}$ The ECG at rest in PPE enhances the power of diagnostic value in athletes with genetic diseases such as cardiomyopathies, channelopathies which are predisposing to sudden cardiac arrest or death. ${ }^{13}{ }^{14}$ In a previous EFSMA statement on ECG for PPE $^{15}$ Löllgen and colleagues presented the pros and cons of having ECG screening integrated in the PPE and concluded that ECG is very sensitive in cardiac screening, and the specificity is increasing constantly, as more studies emerge. Cardiac complications comprise rhythm disorders, structural cardiac diseases or other inherited diseases. Some of these demonstrate underlying genetic causes. Guidelines for detecting these diseases during PPE are strongly improved by checklists, mostly based on family history and ECG findings with ECG interpretation supported by a special sports medicine software of most ECG devices. The software-based interpretation of ECG is superior to visual analysis only. ${ }^{1516}$ Furthermore, the superior role of digital supported ECG interpretation over visual approach for screening athletes and physical active people has been demonstrated by two studies. ${ }^{17}$ Automatic evaluation of athletes ECG device based on current criteria, have made the interpretation more reliable than visual alone. ${ }^{1516}$

Potential cardiac disorders with life-threatening arrhythmias or sudden cardiac arrest are listed in box 1 (modified from Löllgen et $a l^{2}$ ). For most of these diseases, checklists have been developed for a better and more reliable diagnosis. These lists can be downloaded from the EFSMA website together with recommendations on genetic testing (www.EFSMA. org). In all athletes, with even the slightest suspicion, these checklists have to be worked off carefully and mandatory in all tall athletes (eg, $>190 \mathrm{~cm}$ height) such as basketball or volleyball players and rowing athletes, as these athletes often have abortive or manifest signs of Marfan-syndrome. 


\section{WHAT SHOULD BE INCLUDED IN THE PPE?}

The PPE is a medical and legal document developed following a standardised periodic evaluation performed by SEM doctors. It is a complex assessment of the health status, growth and development process and performance level. The SEM doctor assumes responsibility for signing off the PPE, stating that the person can practise a sports activity with the maximal intensity and duration for himself without any contraindications and participate in competitions at the levels indicated. Other important objectives of PPE are to identify factors that may affect performance and factors that increase the risk of future illness and ensure an appropriate medical treatment following antidoping regulation. ${ }^{18}$ At the same time, PPE has a great contribution to the scientific management of sports activity to maintain the well-being and health of the athletes.

The PPE should entail the following diagnostic components:

- Health status.

- Anthropometry.

- Functional and exercise capacity.

\section{Health status}

Health assessment is based on family medical history, personal medical history, training history, training information and medical complaints: symptoms and signs. Standardised history and clinical examination with digital or 'e-documentation' are recommended, storing or paper documentation should be implicit avoided. Establishing the objective health status by physical examination includes the evaluation of dermatological conditions, lymph nodes, musculoskeletal system, respiratory system, cardiovascular system and resting ECG with 12 leads digital recording and software supported. Abdominal, neurological, ophthalmological and ENT examinations, blood and biochemical tests, urine samples should also be assessed. Important goals of PPE are early detection and prevention of severe complications during sports activities in both leisure time and competitive sports. $^{2-5}$

The athlete's history forms have been listed above. ${ }^{1}$ The needed information consists of the year when the athlete started with sports, current type of sports, and results he obtained in competitions, and types of training the athlete performs. It is also important to hear the athlete's goals in sports to provide optimal opportunities and recommendations for achieving peak performance. Further, particular, monitoring training effects of potential difficulties, injuries or overtraining should be asked for in order to get medical counselling or advice.

The physical examination of PPE is very important and it should be performed with a particular focus on cardiovascular, pulmonary, musculoskeletal, neurological, ophthalmological and otolaryngology screening. A thorough neurological assessment is necessary if the athlete has a history of concussion, seizure disorder, cervical spine stenosis or spinal cord injury. This is of special significance for soccer players and rugby or American football to evaluate the risk of cerebral harms.

The musculoskeletal manual screening combines both history and physical examination, to be informed about previous injuries in asymptomatic athletes. The clinician can detect more than $90 \%$ of significant musculoskeletal injuries. The physical screening examination is $51 \%$ sensitive and $97 \%$ specific. So the physician can obtain whether the player has had either a previous injury or even signs or symptoms of injury (eg, pain or tenderness; asymmetric muscle bulk, strength or range of motion; or any obvious deformity). This can be detected during the examination or history, the relevant elements of a site-specific examination should be performed. ${ }^{19}$ It is essential to identify current or past injuries and possible sequelae to determine the athlete's possible risk factors in the future and preventative programmes may be recommended. ${ }^{20}$

Cardiac complications comprise rhythm and cardiac structural abnormalities and inborn diseases such as Marfan-syndrome (see above). A comprehensive medical history, a proper clinical examination alongside a resting ECG can detect occult or congenital diseases that may be life-threatening or disabling.

ECG interpretation requires excellent education in and knowledge of the physiological adaptations of athlete's heart and in deep knowledge of the abnormalities suggestive of a disorder. Current international criteria for ECG interpretation expanded the legitimacy and validity of the results: the rate of false-positive and negative discoveries declining significantly. ${ }^{3}$ International sports medicine and cardiology societies endorsed the international criteria for ECG interpretation in athletes. ${ }^{21}$

The digitalised ECG recordings with software support based on the international criteria are highly sensitive and specific, increasing ECG interpretation's validity. The European Association of Preventive Cardiology recently released a position paper concerning the preparticipation cardiovascular evaluation in athletes ${ }^{21}$ and several consensus papers with recommendations for participation in competitive and leisure time sports in athletes with cardiac diseases. ${ }^{522} 23$

While interpreting the ECG, physicians should look out for any rhythm or conduction abnormalities, QRS morphology, abnormal axis deviation, alteration of the repolarisation and atrial enlargement. The international criteria are very helpful when it comes to deciding which alteration of the ECG is training-related and does not require further investigation.

For the athletes with cardiac abnormalities on the ECG, a multidisciplinary team (cardiology, arrhythmology, cardiac surgery) is more than helpful to decide the most appropriate case management.

Echocardiography allows the physician to sensitively and specifically distinguish adaptive from maladaptive remodelling. Admittedly, structural changes can be missed on the ECG but can be diagnosed precisely through two-dimensional echocardiography including 


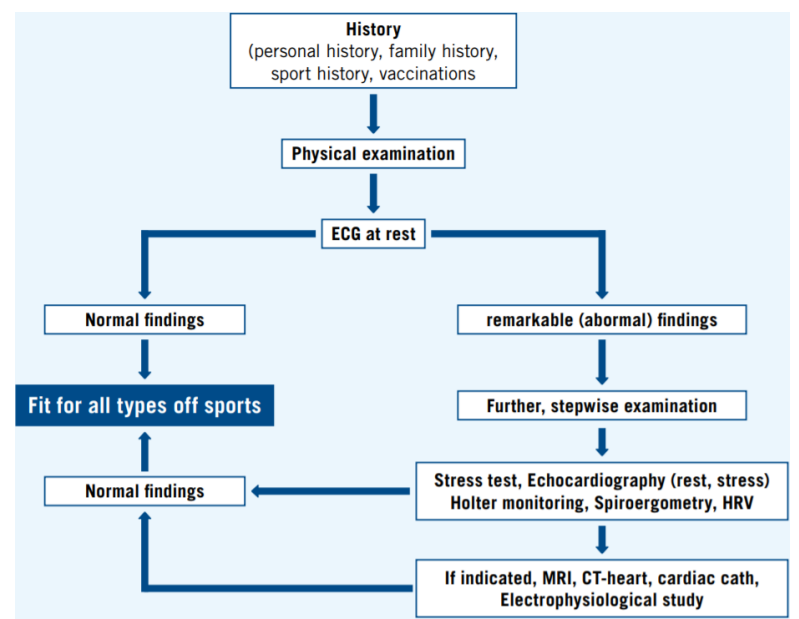

Figure 1 Flow chart of preparticipation examination as recommended by EFSMA. ${ }^{15} \mathrm{HRV}$, heart rate variability.

speckle tracking, strain and stress measurement. Accordingly, an ECG followed by echocardiography would raise the sensitivity and specificity of detecting underlying heart disease.

Transthoracic echocardiography (TTE) allows to evaluate and characterise cardiac morphology and function-very important in assessment of cardiovascular system/conditions. ${ }^{24}$ In the majority of cases, TTE is a second- level examination, preceded by physical examination and rest ECG as shown in the figure 1. Echocardiography is not mandatory for screening. It could be recommended for the first PPE for elite athletes when there is a justified clinical suspicion (eg, suspect ECG findings) with regard to the sports discipline with higher cardiac risk active (high static, high dynamic components).

Echocardiography is performed in athletes:

- In search of pathologies that are a manifestation of cardiovascular diseases that may cause a risk of sudden death.

- In the assessment of the physiological adaptation of the cardiovascular system to physical training and exercises and to differentiate this from pathological findings.

Indication for performing a TTE examination are only after information obtained from clinical history, physical examination and ECG at rest and during exercise test. ${ }^{3}$

Cardiac MRI (CMRI) is very useful tool for the assessment of cardiac structure, function, identify oedema, inflammation and fibrosis in myocardium. This diagnostic tool is used in the case of abnormal history, clinical examination, ECG and usually after echocardiogram. ${ }^{3}$ Today, the SARS-CoV-2 pandemic is more often an indication for CMRI in athletes to detect asymptomatic myocarditis.

The most common indication to CMRI in SEM praxis $\operatorname{are}^{31425-27}$ :

- Differentiation of the athlete's heart with hypertrophic cardiomyopathy, arrhythmogenic cardiomyopathy, dilated cardiomyopathy and left ventricular non-compaction cardiomyopathy.
- Suspicion of active or past myocarditis.

- Coronary artery disease and coronary anomalies.

- Diagnostics of the bicuspid aortic valve.

- Ventricular arrhythmia in athletes with normal TTEto identify spots of myocardial fibrosis.

CMRI has big potential for both clarifying difficult clinical questions and better understanding of the structure and function and diseases of the athlete's heart.

Besides that, additional blood (haematological and biochemical tests depending on the athlete's age and level of training) and urine samples are collected. Blood analysis is a useful tool to optimise athlete's health and performance. Certain biomarkers such as vitamin D and ferritin can directly impact athletic performance, hyperthyroidism can also be detected by basal thyroidstimulanting hormone (TSH), while others like cortisol and creatine kinase can provide insights into overtraining and injuryprevention. ${ }^{28}$ Moreover, it would be important for the healthcare professional to perform a comprehensive nutritional assessment and recommend using supplements only when necessary. The most challenging part of using blood analysis is establishing the baseline level of certain markers for each athlete (hormone profile, inflammation markers, muscle damage markers). The athlete blood test panel should include the complete blood cell count and a comprehensive metabolic panel (glucose blood level, liver panel, kidney profile, lipid profile, electrolytes, vitamin D). When the three diagnostic components of the PPE are completed, the SEM doctor can ask for more biomarkers to be tested to make the right diagnosis (if there is a suspicion of nutritional deficiencies, overtraining, relative energy deficiency in sport (RED-S), hormonal imbalances, inefficient recovery after injury, etc). Blood analysis is a dynamic process that requires careful interpretation in a clinical context considering the timing of the testing (off-season, preseason, competition), the workload of a training session and any other change reported by the athlete (menstrual cycle, injury). The goal is to have a wide view of the health and fitness status of the athletes so that the SEM doctor can make a diagnosis and provide recommendations that are tailor-made for the athlete.

\section{Anthropometry}

The next part of the PPE is the anthropometric examination that includes the somatoscopy, the classic method of assessing posture and body development by visual observation of the athlete in the anthropometric framework and biometrics (weight, height, sitting height, body composition, girth measurements, diameters, mobility and strength measurements) to assess body shape, posture, nutrition, body symmetry, harmony in development, compared with the somatic biotype of the sport.

The somatic biotype represents the set of the most representative somatic qualities necessary for the practice of a certain sport. Evaluating the somatic biotype in athletes is particularly important because there are correlations between the somatic biotype and sports performance. 
Anthropometric indices assess the somatic biotype with predominantly genetic determination (stature, proportions of segments, diameters). Indices that change under the influence of environmental factors (bodyweight), through their large variations, change the appearance of the somatic biotype and therefore can only be interpreted by correlation with body composition. From the point of view of the somatic biotype, we have three categories of sports:

- Sports in which the somatic biotype is essential for obtaining performance: basketball, volleyball, athletics, rowing, artistic gymnastics, extreme categories in weightlifting sports.

- Sports in which the somatic biotype is important: handball, fencing, weightlifting, skiing, swimming, rhythmic gymnastics.

- Sports in which the somatic biotype does not matter: bowling, shooting, chess.

Physicians can also focus on movement quality. This involves identification and rating functional compensations, asymmetries, impairments or efficiency of movement control through transitional or dynamic movement. Examination of the postural alignments of the athletes creates specific compensative or preventive training information to increase athletic performance and prevent sports injuries. ${ }^{29}$

Body composition is the main fitness component for reaching an optimal body shape for the respective type of sport. The body composition assessment is used as a performance or selection criterion, so technique accuracy and reliability are of extreme importance. The body fat varies with age and sex, and the desired body fat of athletes can vary depending on the kind of sport, training level and energy intake. Moreover, the somatic determinants should be correlated with the nutritional status of the athlete to achieve a proper energy intake.

In the process of talent selection and orientation, the anthropometric measurements and abilities of the athlete are also important factors for performance estimation.

\section{Functional and exercise capacity}

The next component of PPE is evaluating functional and exercise capacity according to the predominant type of effort of the sports discipline investigated. This includes neuromuscular evaluation (determination of muscle fibre strength and driving speed, EMG, EEG and Sport Concussion Assessment Tool in contact sports), psychological examination (testing aptitude, adaptation to training and personality traits). Mental health status is vital to discover any obstacles to achieving performance. SEM doctors alongside with sport psychologists can help athletes develop a range of self-management skills that they can use to manage psychological distress.

In competitive athletes, especially top-athletes, PPE without cardio-pulmonary exercise test (CPET) is incomplete. Baseline evaluations can miss important signs and symptoms, which only occur during specific exercise (such as fatigue, functional or non-functional over-reaching and overtraining syndrome or arrhythmias). Managing the issues as mentioned above is very important, and a specialised and educated physician must monitor the interventions required. CPET is the golden standard to monitor the integrative response for exercise capacity, indirect lactate threshold determination, quantification of training management and evaluation via spiroergometry the cardio-respiratory fitness.

The evaluation by CPET allows a complex evaluation of exercise capacity through measurement of gas exchange, pulmonary ventilation response, in addition to blood pressure and heart rate measurement, and exercise ECG (see table 1). This comprehensive method provides an amount of information with regard to energy expenditure and the to many metabolic processes.

The growing volume of evidence supports the use of CPET in the PPE of athletes and diagnosis of possible cardiovascular or pulmonary impairments in the presence of early pathological fatigue and exercise intolerance. It can provide a baseline physical performance assessment before the initiation of a training regimen. It can help establish the intensity of training, assess the performance capacity and evaluate recovery. CPET is a vital tool in SEM to determine which athletes may be presenting a pathology, diagnose latent cardiopulmonary diseases and assess fitness and endurance before or after specific exercise regimens.

Further, evaluation of exercise capacity is an important goal for reaching optimal performance in sports. It allows analysis of athletes' adaptation to the specific effort, establishing the existing limits, the individual specific target heart rates and unveiling how the athletes can push their boundaries. The information following these tests is of great interest to support the work of coaches to achieve maximal performance at the desired time and preventing overtraining syndrome.

Table 1 Parameters to be measured during cardio-pulmonary exercise test

\begin{tabular}{ll}
\hline $\mathrm{VO}_{2}$ max, $\mathrm{VO}_{2}$ max/kg & Ventilator equivalents for $\mathrm{O}_{2}$ and carbon $\mathrm{CO}_{2}$, end-tidal $\mathrm{CO}_{2}$ \\
$\mathrm{HR}$ max, heart rate reserve, BP max & Ventilators thresholds $-\mathrm{VT} 1, \mathrm{VT} 2, \mathrm{VT} 3$ \\
$\mathrm{O}_{2}$ pulse, oxygen saturation & Ventilatory efficiency \\
Breathing equivalent & Respiratory exchange ratio \\
Dead space ventilation, breathing reserve & Respiratory coefficient \\
\hline
\end{tabular}

$\mathrm{BP}$, blood pressure; HR, heart rate. 
As a result, after these evaluations, the athletes receive oral or written recommendations related to their health status, anthropometric measurements, exercise capacity, nutrition, recovery, medication. The athlete needs information, if additional investigations are required to clearly establish a correct diagnosis or correct certain parameters outside the physiological limits.

After the results of the PPE are analysed, athletes will likely get one of the following conclusions:

- Medically eligible for sports performance without restriction.

Medically eligible with recommendation for further evaluation or treatment.

- Not medically eligible for sport performance.

In elite athletes, it is implicit recommended to discuss the result of the PPE with the athlete and, while bearing in mind the need to respect privacy, with the team staff (team doctor, coach, athletic trainer). The team in charge of the athlete needs to create an individualised training plan to achieve performance and anticipate barriers and problems arising along the way. Efforts should be made to have more medical personnel trained to perform a comprehensive PPE (ECG included) and formulate policies to enforce PPE as a key requirement for sports participation. In this way, engaging and receiving input from all stakeholders involved in sports is mandatory.

\section{POSSIBLE BARRIERS IN IMPLEMENTATION OF PPE IN EUROPE}

Preparticipation clinical screening and examination before exercise and competition represents one of the scopes of SEM. However, taking into consideration that SEM is a multidisciplinary specialty there are countries within Europe where SEM does not stand as medical specialty, but as an additional competence. As a result, athletes' screening programmes may differ due to a lack of common specific training for SEM professionals.

The main rationale for EFSMA working group was to create a uniform PPE for all EFSMA sports medicine associations. The multidisciplinary approach of the SEM team is to coordinate and planning of the athlete's health. Nevertheless, all the decision-makers at the national and European level should be involved in the process of implementing the EFSMA recommended PPE. There is a need to work alongside local health authorities and to liaise with both public and private sector so as to put into practice the PPE among all European countries.

Training in ECG interpretation in athletes as well as special ECG devices with programme dedicated to athletes, are mandatory for correct ECG interpretation. Even cardiologists have imperfect expertise in interpretation of athlete's ECG. Appropriate use of standardised criteria of physiological adaptation in bioelectrical function of athlete's heart and knowledge about abnormal ECG findings in this group of people (requiring further evaluation) are the basis of PPE. ${ }^{7140}$ Therefore, training both SEM physicians and cardiologists in athletes' ECG interpretations could represent a challenge for including ECG screening in PPE as recommended by EFSMA. This could be easily overcome by creating more continuous education programmes on this topic.

\section{FUTURE DIRECTIONS}

Nowadays, many healthcare policies focus on decreasing the cost of patient hospitalisation for non-communicable diseases. Therefore, it is imperative that healthcare providers involved in Sport and Exercise Medicine work towards creating a standard of care for athletes of all levels, a standardised digital PPE at among European countries. In addition, there needs to be a focus on PPE educational programmes to have well trained medical personnel and create a well-informed community of athletes. With a complete screening programme, the standardised PPE would promote sports-related and health-related fitness, improve training efficacy, and provide insight into how athletes are responding to stresses occurred during and outside of training and competition. Other benefits of using PPE include gathering scientific explanations for changes in performance or injury risk, enhancing coaches' confidence when manipulating training loads, and improving athlete-coach-SEM physician relationship.

\section{CONCLUSION}

SEM plays an increasingly important role in supporting athletic performance. By developing preventive strategies such as the PPE, we can identify potential health issues at the earliest possible stage and take action accordingly. The screening of athletes through a standardised digital PPE can be also beneficial for the selection process and a better understanding of the impact of long-term physical activity on athletes. Furthermore, a standardised PPE helps the scientific community work closer together in the interest of the athletes.

An easy-to-use electronic PPE with a centralised database allows for international reporting and tracking, with integrated research questions that can provide an overview of athletes' health correlated with their performance. ${ }^{31}$

Once it is established as a European, comprehensive and standardised tool for assessing athletes' readiness to play, it could also be used for conducting research and creating policies for athletes' preparticipation. Therefore, it would be a major step towards improving athletes' health and performance in the medium and long term and a significant contribution to the field.

\section{Author affiliations}

${ }^{1}$ European Federation of Sports Medicine Associations, Lausanne, Switzerland ${ }^{2}$ Sports Medicine, Carol Davila University of Medicine and Pharmacy, Bucuresti, Romania

${ }^{3}$ Collaborating Centre of Sports Medicine, University of Brighton, Eastbourne, UK ${ }^{4}$ Sports Medicine and Rehabilitation Centre, Riga Stradins University, Riga, Latvia

${ }^{5}$ Union Cycliste Internationale, Aigle, Switzerland

${ }^{6}$ Practice for Cardiology, Sports Medicine, Remscheid, Germany

${ }^{7}$ Austrian Institute of Sports Medicine, Vienna, Austria

${ }^{8}$ Sports Medicine Unit, University of Rome Foro Italico, Rome, Italy

${ }^{9}$ Italian Sports Medicine Federation, Rome, Italy

${ }^{10}$ Sports Medicine, Medical University of Lodz, Lodz, Poland 
${ }^{11}$ Lower Limbs- ADMR Hip \& Groin, Defence Medical Rehabilitation Centre Headley Court, Loughborought, UK

Contributors All listed authors have made substantial contributions to the conception or design of the work, or the acquisition/analysis of data or interpretation of the current situation of Sports and Exercise Medicine in Europe. Also, all authors have been involved in drafting or revising the manuscript. Nonetheless, they have approved the final version of the manuscript to be published.

Funding The authors have not declared a specific grant for this research from any funding agency in the public, commercial or not-for-profit sectors.

Competing interests None declared.

Patient consent for publication Not applicable.

Provenance and peer review Not commissioned; externally peer reviewed.

Data availability statement Data sharing not applicable as no datasets generated and/or analysed for this study.

Open access This is an open access article distributed in accordance with the Creative Commons Attribution Non Commercial (CC BY-NC 4.0) license, which permits others to distribute, remix, adapt, build upon this work non-commercially, and license their derivative works on different terms, provided the original work is properly cited, appropriate credit is given, any changes made indicated, and the use is non-commercial. See: http://creativecommons.org/licenses/by-nc/4.0/.

\section{ORCID iDs}

Yannis P Pitsiladis http://orcid.org/0000-0001-6210-2449

Alina Maria Smaranda http://orcid.org/0000-0002-8610-2627

\section{REFERENCES}

1 EFSMA. EFSMA Pre-Participation evaluation (PPE) forms. Available: https://www.efsma.org/newsevents/news-events/251-efsma-preparticipation-evaluations-ppe-forms.html [Accessed 06 Aug 2021]

2 Löllgen H, Löllgen R. Genetics, genetic testing and sports: aspects from sports cardiology. Life Sci Soc Policy 2012;8:32.

3 Pelliccia A, Heidbuchel H, Corrado D. The ESC textbook of sports cardiology. Oxford, UK: Oxford University Press, 2019.

4 Miller SM, Peterson AR. The sports Preparticipation evaluation. Pediatr Rev 2019;40:108-28.

5 Pelliccia A, Solberg EE, Papadakis M, et al. Recommendations for participation in competitive and leisure time sport in athletes with cardiomyopathies, myocarditis, and pericarditis: position statement of the sport cardiology section of the European association of preventive cardiology (EAPC). Eur Heart J 2019;40:19-33.

6 Maron BJ, Friedman RA, Kligfield P. Assessment of the 12-lead $E C G$ as a screening test for detection of cardiovascular disease in healthy general populations of young people (12-25 years of age): a scientific statement from the American heart association and the American College of cardiology. Circulation 2014;130:1303-34.

7 Drezner JA, O'Connor FG, Harmon KG, et al. AMSSM position statement on cardiovascular Preparticipation screening in athletes: current evidence, knowledge gaps, recommendations and future directions. Br J Sports Med 2017;51:153-67.

8 McKinney J, Johri AM, Poirier P, et al. Canadian cardiovascular Society cardiovascular screening of competitive athletes: the utility of the screening electrocardiogram to predict sudden cardiac death. Can J Cardiol 2019;35:1557-66.

9 Drezner JA, Ashley E, Baggish AL, et al. Abnormal electrocardiographic findings in athletes: recognising changes suggestive of cardiomyopathy. Br J Sports Med 2013;47:137-52.

10 Drezner JA, Ackerman MJ, Cannon BC, et al. Abnormal electrocardiographic findings in athletes: recognising changes suggestive of primary electrical disease. $\mathrm{Br} J$ Sports Med 2013;47:153-67.

11 Exeter DJ, Elley CR, Fulcher ML, et al. Standardised criteria improve accuracy of ECG interpretation in competitive athletes: a randomised controlled trial. Br J Sports Med 2014;48:1167-71.
12 Drezner JA, Ackerman MJ, Anderson J, et al. Electrocardiographic interpretation in athletes: the 'Seattle criteria'. Br J Sports Med 2013;47:122-4

13 Harmon KG, Zigman M, Drezner JA. The effectiveness of screening history, physical exam, and ECG to detect potentially lethal cardiac disorders in athletes: a systematic review/meta-analysis. $J$ Electrocardiol 2015;48:329-38.

14 Mont L, Pelliccia A, Sharma S, et al. Pre-participation cardiovascular evaluation for athletic participants to prevent sudden death: position paper from the EHRA and the EACPR, branches of the ESC. endorsed by APHRS, HRS, and SOLAECE. Eur J Prev Cardiol 2017;24:41-69.

15 Löllgen H, Börjesson M, Cummiskey J, et al. The Pre-Participation examination in sports: EFSMA statement on ECG for PreParticipation examination. Dtsch Z Sportmed 2015;2015:151-5.

16 Berge HM, Steine K, Andersen TE, et al. Visual or computer-based measurements: important for interpretation of athletes' ECG. Br J Sports Med 2014;48:761-7.

17 Abächerli R, Schmid R, Richard K, et al. OC06_06 Automatic Implementation of the Athlete's Seattle Criteria Leads to Less than Ten Percent of Abnormal Electrocardiograms in General Young Population of Swiss Males. Glob Heart 2016;11:(Suppl):Pe8.

18 Bernhardt DT, Roberts WO. Ppe: Preparticipation physical evaluation. American Academy of Pediatrics 2019.

19 Conley KM, Bolin DJ, Carek PJ, et al. National athletic trainers' association position statement: Preparticipation physical examinations and disqualifying conditions. J Athl Train 2014:49:102-20.

20 Pruna R, Lizarraga A, Domínguez D. Medical assessment in athletes. Med Clin 2018;150:268-74

21 Drezner JA, Sharma S, Baggish A, et al. International criteria for electrocardiographic interpretation in athletes: consensus statement. Br J Sports Med 2017;51:704-31.

22 Niebauer J, Börjesson M, Carre F, et al. Recommendations for participation in competitive sports of athletes with arterial hypertension: a position statement from the sports cardiology section of the European association of preventive cardiology (EAPC). Eur Heart J 2018;39:3664-71.

23 Borjesson M, Dellborg M, Niebauer J, et al. Recommendations for participation in leisure time or competitive sports in athletespatients with coronary artery disease: a position statement from the sports cardiology section of the European association of preventive cardiology (EAPC). Eur Heart J 2019:40:13-18.

24 Steeds RP, Garbi M, Cardim N, et al. EACVI appropriateness criteria for the use of transthoracic echocardiography in adults: a report of literature and current practice review. Eur Heart J Cardiovasc Imaging 2017;18:1191-204

25 Androulakis E, Swoboda PP. The role of cardiovascular magnetic resonance in sports cardiology; current utility and future perspectives. Curr Treat Options Cardiovasc Med 2018;20:86.

26 Pelliccia A, Caselli S, Sharma S, et al. European Association of Preventive Cardiology (EAPC) and European Association of Cardiovascular Imaging (EACVI) joint position statement: recommendations for the indication and interpretation of cardiovascular imaging in the evaluation of the athlete's heart. Eur Heart J 2018;39:1949-69.

27 Galderisi M, Cardim N, D'Andrea A, et al. The multi-modality cardiac imaging approach to the Athlete's heart: an expert consensus of the European Association of Cardiovascular Imaging. Eur Heart $J$ Cardiovasc Imaging 2015;16:353-353r

28 Lee EC, Fragala MS, Kavouras SA, et al. Biomarkers in Sports and Exercise: Tracking Health, Performance, and Recovery in Athletes. $J$ Strength Cond Res 2017;31:2920-37.

29 Toivo K, Kannus P, Kokko S, et al. Musculoskeletal examination in young athletes and non-athletes: the Finnish health promoting sports Club (FHPSC) study. BMJ Open Sport Exerc Med 2018;4:e000376.

30 Sharma S, Drezner JA, Baggish A, et al. International recommendations for electrocardiographic interpretation in athletes. Eur Heart J 2018;39:1466-80.

31 Roberts WO, Löllgen H, Matheson GO, et al. Advancing the preparticipation physical evaluation: an ACSM and FIMS joint consensus statement. Clin J Sport Med 2014;24:442-7. 